\title{
Integrative analysis of vascular endothelial cell genomic features identifies AIDA as a coronary artery disease candidate gene
}

Simon Lalonde ${ }^{1 \dagger}$, Valérie-Anne Codina-Fauteux ${ }^{1,2 \dagger}$, Sébastian Méric de Bellefon ${ }^{1,2}$, Francis Leblanc ${ }^{1,2}$, Mélissa Beaudoin ${ }^{1}$, Marie-Michelle Simon ${ }^{3}$, Rola Dali ${ }^{3}$, Tony Kwan ${ }^{3}$, Ken Sin Lo ${ }^{1}$, Tomi Pastinen ${ }^{4}$ and Guillaume Lettre $e^{1,2^{*}}$ (D)

\begin{abstract}
Background: Genome-wide association studies (GWAS) have identified hundreds of loci associated with coronary artery disease (CAD) and blood pressure (BP) or hypertension. Many of these loci are not linked to traditional risk factors, nor do they include obvious candidate genes, complicating their functional characterization. We hypothesize that many GWAS loci associated with vascular diseases modulate endothelial functions. Endothelial cells play critical roles in regulating vascular homeostasis, such as roles in forming a selective barrier, inflammation, hemostasis, and vascular tone, and endothelial dysfunction is a hallmark of atherosclerosis and hypertension. To test this hypothesis, we generate an integrated map of gene expression, open chromatin region, and 3D interactions in resting and TNFatreated human endothelial cells.
\end{abstract}

Results: We show that genetic variants associated with CAD and BP are enriched in open chromatin regions identified in endothelial cells. We identify physical loops by Hi-C and link open chromatin peaks that include CAD or BP SNPS with the promoters of genes expressed in endothelial cells. This analysis highlights 991 combinations of open chromatin regions and gene promoters that map to 38 CAD and 92 BP GWAS loci. We validate one CAD locus, by engineering a deletion of the TNFa-sensitive regulatory element using CRISPR/Cas9 and measure the effect on the expression of the novel CAD candidate gene AIDA.

Conclusions: Our data support an important role played by genetic variants acting in the vascular endothelium to modulate inter-individual risk in CAD and hypertension.

Keywords: Vascular endothelium, Endothelial dysfunction, Coronary artery disease, Blood pressure, Hypertension, Genome-wide association study, Hi-C, AIDA, CRISPR/Cas9

\section{Background}

Genetic discoveries in humans have the potential to unravel novel pathophysiological mechanisms and to pinpoint promising drug targets [1]. However, to meet our expectations, these discoveries ought to be supported by mechanistic studies to decipher how genetic variation

\footnotetext{
*Correspondence: guillaume.lettre@umontreal.ca

${ }^{\dagger}$ Simon Lalonde and Valérie-Anne Codina-Fauteux contributed equally to this work.

'Montreal Heart Institute, 5000 Belanger street, Montréal, Québec H1T 1C8, Canada

Faculté de Médecine, Université de Montréal, Montréal, Québec H3T 1J4, Canada

Full list of author information is available at the end of the article
}

modulates disease risk. For genome-wide association study (GWAS) discoveries, the design of such functional experiments is particularly challenging as the vast majority of the associated variants are non-coding. Furthermore, we often ignore in which organ(s) or cell type(s) the variants act. Methods have been developed by which we can quantify the enrichment of GWAS variants within regulatory elements identified by transcriptomic or epigenomic profiling of human samples [2-4]. Although powerful, such methods remain probabilistic and further experiments are required to test their predictions. As a consequence, only a few association signals have been resolved at the molecular level [5-7].

(c) The Author(s). 2019 Open Access This article is distributed under the terms of the Creative Commons Attribution 4.0 International License (http://creativecommons.org/licenses/by/4.0/), which permits unrestricted use, distribution, and 
GWAS have identified hundreds of variants associated with coronary artery disease (CAD) [8-10] and blood pressure (BP) or hypertension $[11,12]$. Many of these association signals implicate excellent candidate genes and independently confirm some of the biology previously known to influence these diseases, such as the role that blood lipid levels play in CAD risk or the importance of smooth muscle contraction in controlling BP. But for many loci, we ignore how they might contribute to the development of these diseases, either because there are no obvious candidate genes nearby or because the variants are not associated with known risk factors. For instance, for CAD, it is estimated that nearly half of the 140 loci identified by GWAS do not associate with the traditional risk factors (e.g., blood lipids, type 2 diabetes, blood pressure) [13].

Annotation of GWAS discoveries for CAD and BP has revealed an enrichment of associated variants near genes implicated in endothelial functions [9, 12]. Vascular endothelial cells form the inner layer of blood vessels and play a critical role in the etiology of CAD and hypertension. Indeed, healthy endothelial cells form a selective barrier between the blood and the intima for many macromolecules, respond to hemodynamic changes, control the vascular tone, and regulate platelet functions, inflammatory responses, and smooth muscle cell growth and migration [14]. Despite their pathophysiological importance and the noted overlap with GWAS findings, endothelial cells have not been studied extensively to provide further insights into genotypephenotype associations for $\mathrm{CAD}$ and $\mathrm{BP} /$ hypertension. Here, we profiled the transcriptome, epigenome, and 3D chromosome conformation of vascular endothelial cells and integrate these results with CAD- and BP-associated genetic variants. Because the effect of genetic variation can be specific to certain pathological states [15], we characterized not only resting endothelial cells, but also cells activated with the inflammatory cytokine tumor necrosis factor- $\alpha$ (TNF $\alpha)$. Finally, we used our datasets to generate mechanistic hypotheses and tested one such prediction at a CAD locus using the CRISPR/Cas9 genome editing system.

\section{Results}

Transcriptomic and epigenomic changes in endothelial cells upon activation

To develop a tractable endothelial cellular system to study the molecular mechanisms that contribute to the etiology of vascular diseases such as atherosclerosis and hypertension, we characterized the response of teloHAEC to the potent pro-inflammatory cytokine TNFa. We selected TNF $\alpha$ to activate teloHAEC because it generates a very robust and reproducible inflammatory response [16]. TeloHAEC are immortalized human aortic endothelial cells with many of the cardinal features of endothelial cells (e.g., expression of cell surface marker (CD31/PECAM1), angiogenesis potential, LDL-cholesterol uptake) and a normal karyotype (46,XX; confirmed by cytogenetics and whole-genome DNA sequencing). We treated teloHAEC with TNF $\alpha$ for 4 and $24 \mathrm{~h}$ and compared gene expression levels between unstimulated (NT) and stimulated cells by RNA-sequencing (RNAseq). In total when considering all replicates and timepoints, we identified 1316 differentially expressed genes (false discovery rate $(\mathrm{FDR})<0.1 \%$ and absolute $\log _{10}$ fold-change $(|\mathrm{LFC}|)>0.3$ )(Fig. 1a for the comparison of NT vs. $4 \mathrm{~h}$ TNFo treatment, Additional file 1 for all other comparisons, and Additional file 2 for the complete list of differentially expressed genes). Not surprisingly, many of the most upregulated genes are well-known markers of endothelial dysfunction (e.g., SELE, ICAM1, SOD2, IL8, IL1B) (Additional file 2). Pathway analyses confirmed that most of the transcriptional changes due to TNF $\alpha$ treatment are captured by inflammatory pathways such as TNF $\alpha$ signaling, cytokine-cytokine receptor interaction, and NF- $\mathrm{kB}$ signaling (Additional file 3). In parallel, we also treated primary human coronary artery endothelial cells (HCAEC) with $\mathrm{TNF} \alpha$ and measured transcript levels by RNAseq. The transcriptional response to TNF $\alpha$ stimulation was highly concordant between immortalized teloHAEC and primary HCAEC in all timepoint comparisons (Pearson's $r>0.6, P$ value $<2.2 \times 10^{-16}$, Fig. 1b), suggesting that teloHAEC represents a good cellular model to study vascular endothelial cell activation.

To correlate changes in gene expression with chromatin activity, we also profiled open chromatin regions by Assay for Transposase-Accessible Chromatin using sequencing (ATACseq) in teloHAEC treated or not with TNF $\alpha$ for 4 or $24 \mathrm{~h}$. By combining data from these different time points, we identified 95,491 ATACseq peaks, including 3138 peaks (3.3\%) that are differentially opened or closed (FDR $<0.1 \%$ and $|\mathrm{LFC}|$ ) $>0.3$ ) upon TNF $\alpha$ stimulation (Fig. 1c for the comparison of NT vs. 4h TNF $\alpha$ treatment, Additional file 1 for all other comparisons, and Additional file 4 for the complete list of differentially opened or closed ATACseq peaks). Although results in Fig. 1c seem to indicate that most ATACseq peaks open upon TNF $\alpha$ treatment, a density analysis of these data points shows that most ATACseq peak LFC are centered at 0 (Additional file 5). As for the transcriptional response, the magnitude of open chromatin regions defined by ATACseq was highly concordant between teloHAEC and HCAEC (Fig. 1d). We employed an in silico footprinting method to determine which transcription factor binding motifs are over-represented within differentially opened teloHAEC ATACseq peaks following TNF $\alpha$ treatment (Additional file 6) [17]. Many of these transcription factors are involved in 

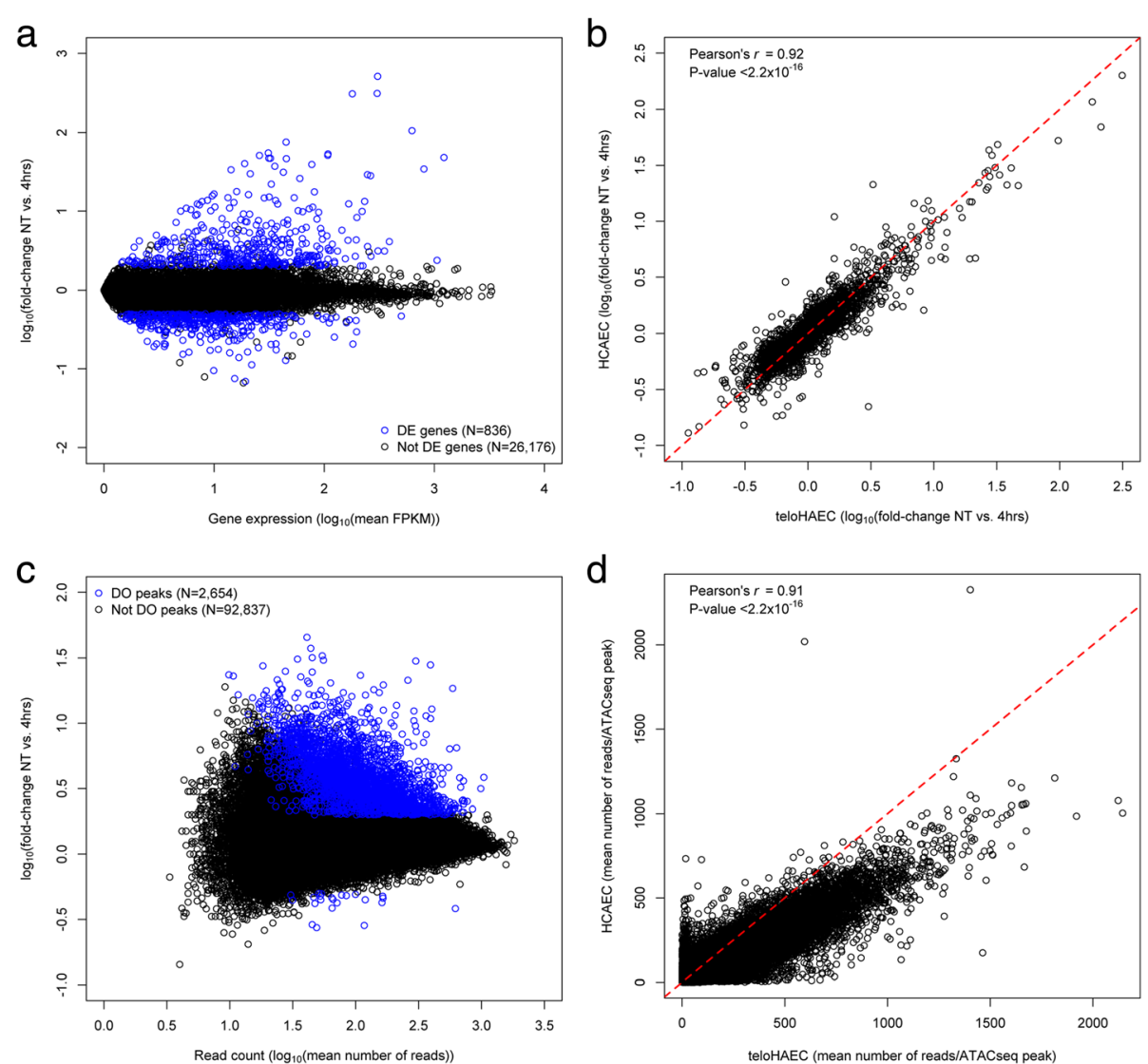

Fig. 1 Transcriptomic and epigenomic profiling of teloHAEC. a RNAseq of teloHAEC non-treated (NT) or treated with TNFa identified 1316 differentially expressed (DE) genes (FDR $<0.1 \%$ and absolute $\log _{10}$-fold-change $>0.3$ ) among three comparisons (NT vs. 4 h, NT vs. 24 h, $4 \mathrm{~h}$ vs. 24 h). Of these 1316 genes, 836 genes were DE in the NT vs. $4 \mathrm{~h}$ comparison. $\mathbf{b}$ Gene expression fold-change for DE genes are highly correlated between transformed teloHAEC and primary HCAEC. All three comparisons are highly significant $\left(P<2.2 \times 10^{-16}\right)$, but for simplicity we only show the NT vs. $4 \mathrm{~h}$ comparison. c ATACseq of teloHAEC NT or treated with TNFa identified 95,491 peaks, including 3138 differentially opened (or closed) (DO) peaks (FDR $<0.1 \%$ and absolute $\log _{10}$-fold-change $>0.3$ ) among three comparisons (NT vs. $4 \mathrm{~h}, \mathrm{NT}$ vs. $24 \mathrm{~h}, 4 \mathrm{~h}$ vs. $24 \mathrm{~h}$ ). Of these 3138 peaks, 2654 peaks were DO in the NT vs. $4 \mathrm{~h}$ comparison. $\mathbf{d}$ Open chromatin regions (raw number of reads), identified by ATACseq, are highly correlated between teloHAEC and HCAEC. Results shown are for the $4 \mathrm{~h}$ TNFa treatment. Results are consistent for the NT and $24 \mathrm{~h}$ timepoints. The distribution falls under the diagonal because the coverage of the ATACseq teloHAEC libraries was higher than the coverage of the HCAEC libraries

inflammatory responses (e.g., JUN, FOS, NFKB1/2) (Additional file 7). To further characterize our ATACseq open chromatin dataset, we generated histone H3 lysine 27 acetylation (H3K27ac) data in NT and TNFo-treated teloHAEC using chromatin immunoprecipitation followed by sequencing (ChIPseq). H3K27ac marks highlight regions of active transcription and are found at enhancers and promoters [18]. Within each condition (NT or with TNFo), we found that $70-74 \%$ of the ATACseq peaks intersected with $\mathrm{H} 3 \mathrm{~K} 27 \mathrm{ac}$ peaks.

Most genetic variation associated with complex human traits by GWAS is in non-coding regions [19, 20]. To evaluate the relevance of our teloHAEC TNF $\alpha$ stimulated system to study vascular diseases, we measured the enrichment of single nucleotide polymorphisms (SNPs) associated with CAD or BP in ATACseq peaks identified in teloHAEC. For comparison, we also retrieved ATACseq data from 27 different tissues from the ENCODE Project. For these analyses, we considered CAD- and BP-associated SNPs (as well as their linkage disequilibrium (LD) proxies) obtained from recent largescale meta-analyses: we tested 175 sentinel (5117 LD proxies) CAD and 357 sentinel (13,970 proxies) BP SNPs $[8,11]$. We also used 97 SNPs (3953 proxies) associated with body mass index (BMI) as control genetic variants not associated with a vascular phenotype [21]. The fraction of ATACseq peaks that included CAD-associated SNPs was similar between teloHAEC and coronary arteries, although the enrichment was higher for the esophagus muscularis mucosa and the right atrium auricular region (Fig. 2). For BP-associated SNPs, teloHAEC had the strongest enrichment when compared to all other tested tissues (Fig. 2). Furthermore, the fraction of teloHAEC ATACseq peaks with CAD or BP SNPs was 


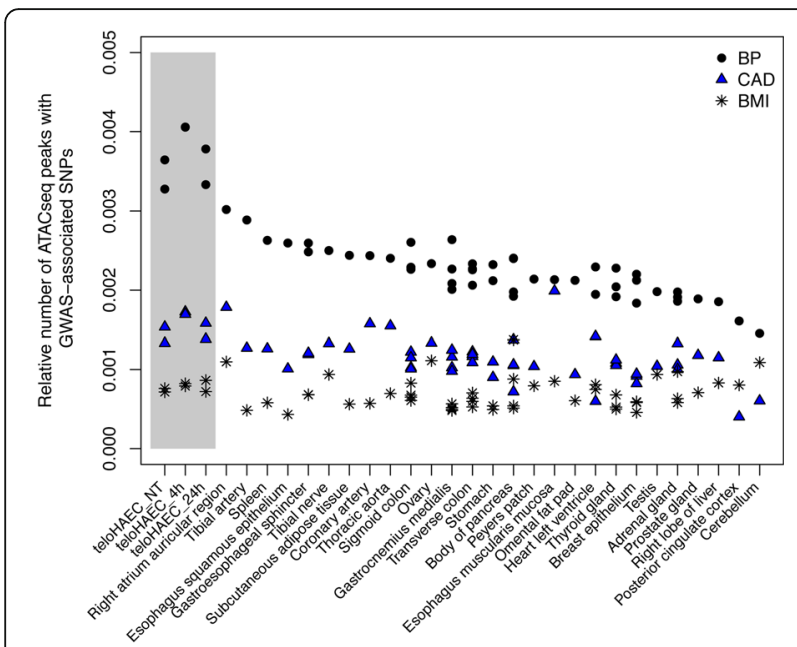

Fig. 2 Enrichment of blood pressure (BP) and coronary artery disease (CAD)-associated SNPs in open chromatin regions. We compared overlap in endothelial cells (teloHAEC) and all available tissues from ENCODE. Each biological replicate is identified by a different point. We called all ATACseq peaks with the same bioinformatic pipeline. To account for the different coverage of each ATACseq library, we present the relative fraction of ATACseq peak that overlap with a BP or CAD SNP. We used body mass index (BMI)-associated SNPs as controls, since BMI is not a vascular phenotype. The gray box highlights results generated in this study in nontreated (NT) or TNFa-stimulated ( 4 or $24 \mathrm{~h}$ ) teloHAEC

higher than for BMI variants (Fig. 2). Enrichments of CAD- and BP-associated SNPs in ATACseq peaks in teloHAEC were highly significant when controlling for the genome-wide distribution of these peaks, although they were not markedly stronger when focusing on differentially opened/closed ATACseq peaks (Additional file 8) [22]. We obtained highly concordant enrichments when we overlapped CAD and BP SNPs with the location of ChIPseq peaks for the histone mark H3K27ac generated in teloHAEC without or with TNF $\alpha$ (Additional file 8). We identified 263 sentinel or proxy SNPs associated with $\mathrm{CAD}$ or $\mathrm{BP}$ that map onto a transcription factor binding motif found in a teloHAEC ATACseq peak (Additional file 9).

\section{D chromosomal architecture in endothelial cells}

One outstanding challenge in gaining biological insights from GWAS discoveries is to connect variants located in non-coding regulatory elements with their target genes. When cells or tissues from many human donors are profiled, it is possible to use the covariance between open chromatin regions and expression levels of nearby genes to infer that connection. As an alternative solution to link genes and regulatory elements in the context of endothelial dysfunction, we generated genomic contact maps by Hi-C using untreated and TNF $\alpha$-stimulated (4 h) teloHAEC. For each condition, the contact matrices were highly concordant across biological replicates
(Pearson's correlation $r>0.95$ for the contact matrices at $10-\mathrm{kb}$ resolution across all replicates), allowing us to combine datasets to increase the signal-to-noise ratios of our analyses.

The genome is divided between active and repressed regions, often referred to as $\mathrm{A}$ and $\mathrm{B}$ compartments [23]. Using principal component analysis on the $\mathrm{Hi}-\mathrm{C}$ datasets, we identified A and B compartments and compared their distribution genome-wide between NT and TNF $\alpha$ stimulated teloHAEC. Compartments were highly correlated between conditions, with only $2.1 \%$ of the genome that switched following exposure to TNF $\alpha$ (Fig. 3a, b). We compared gene expression (RNAseq) and open chromatin region (ATACseq) changes in teloHAEC (NT vs. 4h TNFo) with the A/B switching compartments defined by $\mathrm{Hi}-\mathrm{C}$. Upregulated genes were enriched in Bto-A compartments $(61.4 \%$ of genes in B-to-A compartments are upregulated vs. $43.5 \%$ of all genes expressed upon TNF $\alpha$ treatment; enrichment $=1.4 ; P_{\text {binomial }}=1.5 \times$ $\left.10^{-6}\right)$, and downregulated genes were enriched in A-to-B compartments $(82.9 \%$ of genes in A-to-B compartments are downregulated vs. $56.5 \%$ of all genes expressed upon $\mathrm{TNF} \alpha \quad$ treatment; $\quad$ enrichment $=1.5 ; \quad P_{\text {binomial }}=4.8 \times$ $\left.10^{-6}\right)$ (Fig. 3c). ATACseq peaks with higher coverage (opening chromatin) after TNF $\alpha$ stimulation were overrepresented in B-to-A switching compartments (90.8\% of ATACseq peaks in B-to-A compartments are more open vs. $79.7 \%$ of all ATACseq peaks upon TNF $\alpha$ treatment; enrichment $\left.=1.1 ; P_{\text {binomial }}=7.6 \times 10^{-6}\right) \quad($ Fig. $3 \mathrm{~d})$. We could not detect a significant enrichment of ATACseq peaks with lower coverage (closing chromatin) among A-to-B compartments $\left(P_{\text {binomial }}=0.57\right)($ Fig. $3 \mathrm{~d})$, potentially because there are fewer ATACseq peaks that close after TNF $\alpha$ treatment for $4 \mathrm{~h}$ (Fig. 1c).

Topologically associated domains (TADs) are defined by a high density of interactions between non-adjacent chromosomal regions and represent functional units of genome organization important for gene regulation [24]. We identified 4148 and 4078 TADs in our $\mathrm{Hi}-\mathrm{C}$ data from NT and TNF $\alpha$-stimulated teloHAEC, respectively (Fig. 3b). TADs were highly correlated between NT and TNF $\alpha$-treated endothelial cells, and only $7.7 \%$ of TAD boundaries changed upon TNF $\alpha$ treatment. Previous studies have shown that TAD boundaries are enriched with binding motifs for the transcription factor CTCF and the promoters of expressed genes [23, 25]. To validate the TADs that we identified, we retrieved from the ENCODE Project CTCF ChIPseq data from human umbilical vein endothelial cells (HUVEC) and showed that the ENCODE HUVEC CTCF peaks are enriched at teloHAEC TAD boundaries (Fig. 4a and Additional file 10). We also confirmed that transcriptional start sites (TSSs) defined using our teloHAEC RNAseq data were enriched at TAD boundaries (Fig. 4a and Additional file 10). 

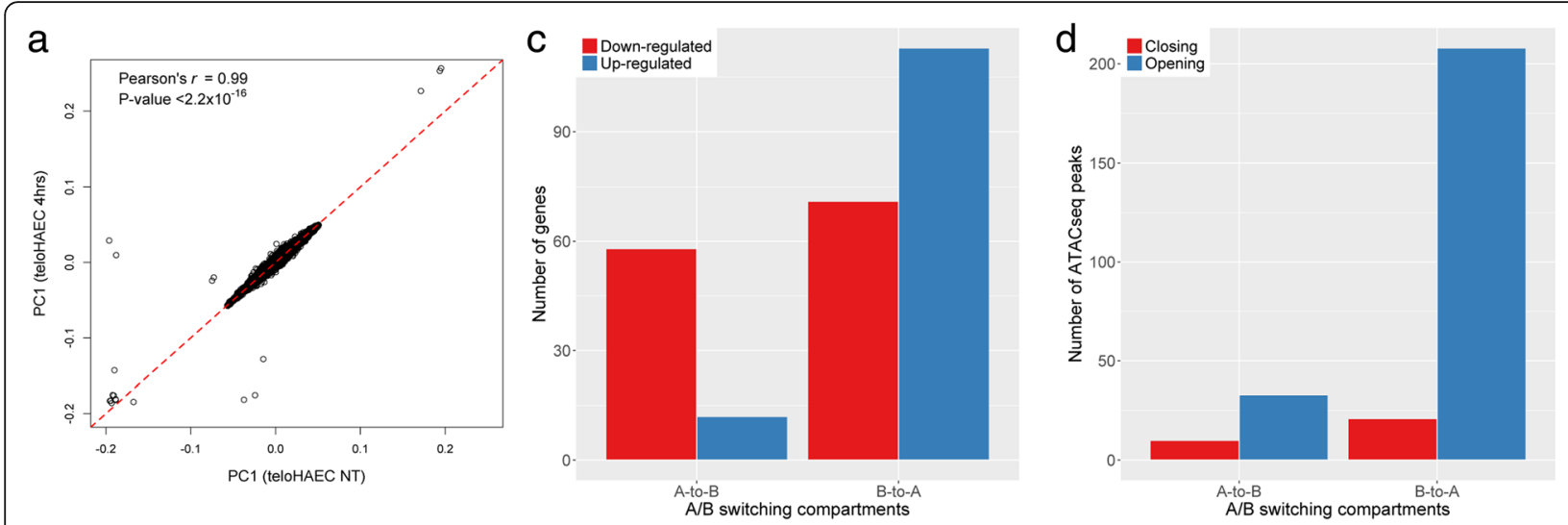

b

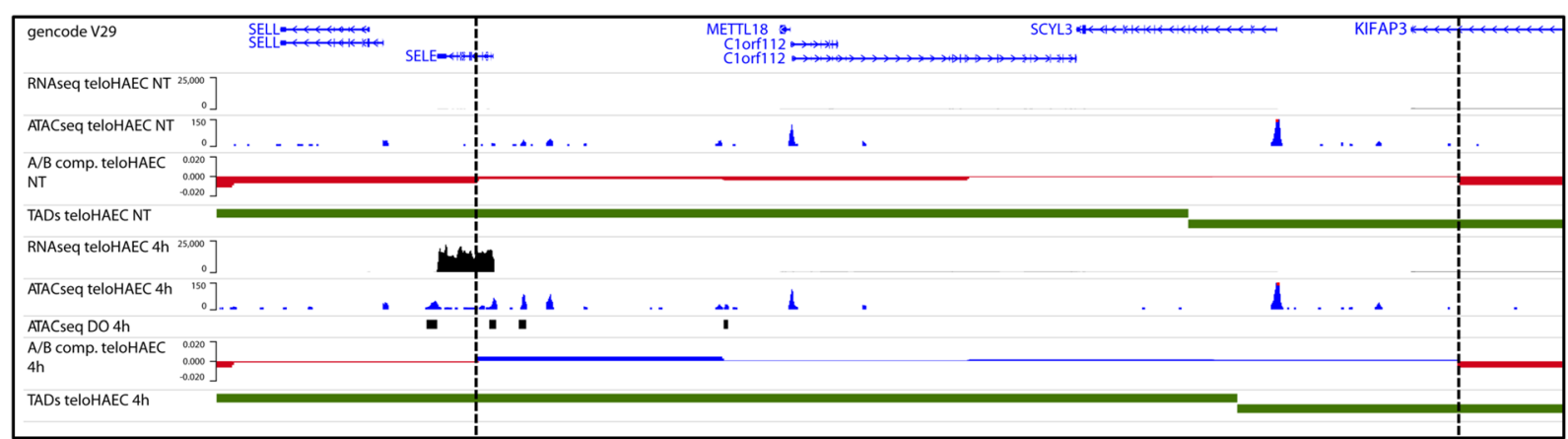

Fig. 3 Gene expression and open chromatin regions in switching A/B compartments. a Correlation of principal component 1 (PC1) calculated on the Hi-C contact matrices from non-treated (NT) or TNFa-stimulated ( $4 \mathrm{~h}$ ) teloHAEC. b B-to-A compartment switch at the E-selectin (SELE) locus on chromosome $1 \mathrm{q} 24$ following TNFa treatment in teloHAEC for $4 \mathrm{~h}$. TNFa treatment strongly induces SELE expression (RNAseq NT vs. $4 \mathrm{~h}$ ) and opens several elements at the locus (ATACseq NT vs. $4 \mathrm{~h}$, differentially opened ATACseq peaks (ATACseq DO $4 \mathrm{~h}$ )). The 2 vertical dashed lines indicate the boundaries of a compartment that switch from the repressed B state in NT teloHAEC (red) to the active A state (blue) after $4 \mathrm{~h}$ of TNFa treatment. c Genes with down-regulated expression after $4 \mathrm{~h}$ of TNFa treatment are enriched in active-to-repressed (A-to-B) switching compartments, whereas up-regulated genes are enriched in B-to-A switching compartments. $\mathbf{d}$ ATACseq peaks that are more opened after TNFa treatment for $4 \mathrm{~h}$ are significantly enriched in B-to-A switching compartments
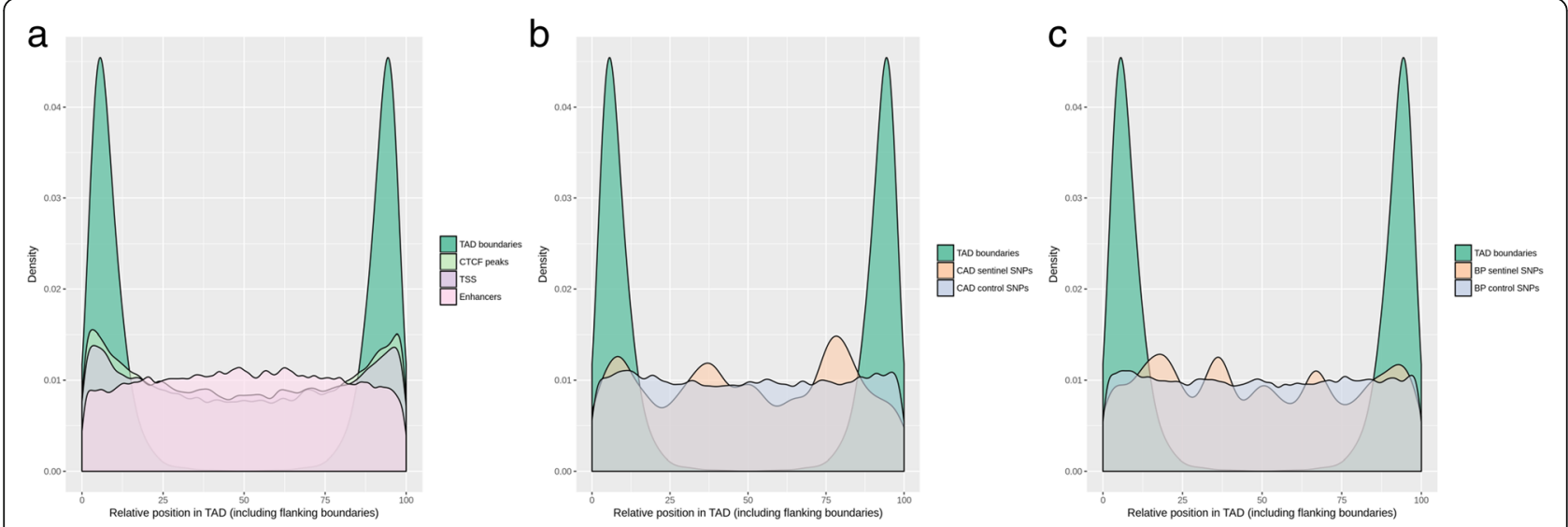

Fig. 4 Topologically associated domains (TADs) in teloHAEC endothelial cells treated with TNFa. a Because TADs have different sizes across the genome, we normalized them after adding $35 \mathrm{~kb}$ on either side to define boundaries. From ENCODE Project data in HUVECS, we retrieved CTCF binding sites from ChIPseq and enhancers defined with histone marks. We used our own RNAseq data in teloHAEC to define transcription start sites (TSS). In $\mathbf{b}$ and $\mathbf{c}$, we map the relative position of coronary artery disease (CAD)- and blood pressure (BP)-associated SNPs into teloHAEC TADs. For comparison, we also added the distribution of relative positions for non-associated, matched (control) SNPs. Similar results were observed for TADs in non-treated teloHAEC (Additional file 10) 
These orthogonal datasets attest to the quality of our Hi-C experiments.

Given the central role that TADs play in the regulation of gene expression, we next asked where within TADs are located ENCODE enhancers predicted by histone marks [18]. In contrast to TSSs, we found that enhancers defined in HUVEC by ENCODE were more uniformly distributed with a slight enrichment in the middle of teloHAEC TADs as opposed to the boundaries (Fig. 4a and Additional file 10). Finally, we mapped CAD- and BP-associated SNPs into TADs and compared their physical distance from the closest TAD boundary with the distance of non-associated matched SNPs. Because of the relatively small number of CAD and BP sentinels SNPs (175 and 357 variants, respectively), the distributions of their position relative to the TAD boundaries were uneven (Fig. 4b, c and Additional file 10). For both CAD and BP, associated SNPs tended to be closer from the nearest TAD boundary than matched SNPs (median distance $75 \mathrm{~kb}$ for associated SNPs vs. $103 \mathrm{~kb}$ for matched SNPs, empirical $P$ values $\leq 0.04$ ), although a larger number of sentinel variants would be needed to provide a definitive answer to this question.

\section{Linking GWAS SNPs and regulatory elements with genes}

We used the Hi-C contact matrices to call loops between regulatory elements that contain $\mathrm{CAD}$ - or $\mathrm{BP}$ associated variants and the promoter of genes expressed in teloHAEC. To further refine this list, we applied several criteria: we considered 3D loops supported by $\geq 20$ $\mathrm{Hi}-\mathrm{C}$ reads, we excluded genes that are not expressed or expressed at low levels (bottom 10 percentile) in teloHAEC, and we prioritized open chromatin regions that contain CAD or BP SNPs that are expression quantitative trait loci (eQTL) for the linked genes in the GTEx dataset $(P$ value $<0.001)[26]$. After filtering, this analysis identified 991 combinations of open chromatin regions and genes linked by physical 3D interactions and eQTL results (Additional files 11, 12, and 13). These combinations map to $38 \mathrm{CAD}$ and $92 \mathrm{BP}$ GWAS loci. The average physical distance between these regulatory elements and gene promoters is $154 \pm 158 \mathrm{~kb}$ (Additional file 11).

We attempted to validate one of our molecular predictions, focusing on interactions where the open chromatin region and the linked gene are modulated by TNF $\alpha$ treatment and where the variant is a strong eQTL (GTEx $P$ value $<1 \times 10^{-5}$ ). Such additional stringent filtering criteria highlighted two combinations of SNP, open chromatin region and gene at the AIDA and TRAF1 loci (Additional file 11). We selected the AIDA locus for further functional characterization because it is associated with CAD; the TRAF1 SNP is associated with BP (Fig. 5a). Using the CRISPR/Cas9 system in teloHAEC, we engineered a 1022 base pair deletion in the
MIA3 gene that contains a TNF $\alpha$-sensitive open chromatin element that physically interacts with the promoter of the differentially expressed gene AIDA. This deletion encompasses rs17163363, a strong proxy for the sentinel CAD SNP rs67180937 $\left(r^{2}=1\right.$ in European populations from the 1000 Genomes Project) that is also an eQTL for AIDA in GTEx esophagus muscularis samples $\left(P_{\mathrm{eQTL}}=1.4 \times 10^{-6}\right)$. Although we could not retrieve homozygous clones for the deletion, we obtained three independent heterozygotes and tested the induction of AIDA expression following TNF $\alpha$ treatment using two different qPCR assays (Fig. 5a and Additional file 14). Whereas TNF $\alpha$ could induce a robust AIDA expression response in cells without a deletion $\left(+29 \%, P_{\mathrm{qPCR} 1}=\right.$ 0.0013 and $P_{\mathrm{qPCR} 2}=0.012$ ), the increase in AIDA expression was roughly half that induction level in heterozygous teloHAEC $\left(+15 \%, \quad P_{\mathrm{qPCR} 1}=0.16\right.$ and $P_{\mathrm{qPCR} 2}=$ 0.026), likely because one allele is still functional (Fig. 5b, c). This result is consistent with our model by which this regulatory element-and presumably the genetic variant(s) that it contains-can control the expression of $A I D A$ only upon endothelial cell activation.

\section{Discussion}

GWAS have identified hundreds of variants robustly associated with $\mathrm{CAD}$ and $\mathrm{BP} /$ hypertension. Despite recent efforts, the causal variants, genes, and tissues/cell types remain largely unknown at these loci. In this study, we tested the hypothesis that some of these genetic associations are mediated through the activity of DNA sequence variants that control gene expression upon vascular endothelial cell activation. We profiled the transcriptome (RNAseq) and open-chromatin genome (ATACseq) of resting and TNF $\alpha$-activated immortalized human aortic endothelial cells (teloHAEC). We focus on these transformed cells in order to develop a system amenable for efficient genome editing experiments, an essential component of any GWAS follow-up program. We confirmed the RNAseq and ATACseq results from teloHAEC in primary human coronary artery endothelial cells. Furthermore, we generated and characterized genome-wide chromosome conformation $\mathrm{Hi}-\mathrm{C}$ contact matrices from NT and TNF $\alpha$-treated teloHAEC cells to physically link regulatory elements and expressed genes. By integrating our results with publicly available epigenomic datasets from ENCODE, eQTL results from GTEx, and GWAS discoveries for CAD and BP, we created a dynamic regulatory map of vascular endothelial cells. Through this map, we identified CAD and BP variants that overlap with open chromatin regions which themselves physically interact with often distant gene promoters in a specific cellular inflammation/non-inflammation context (Additional file 11). 

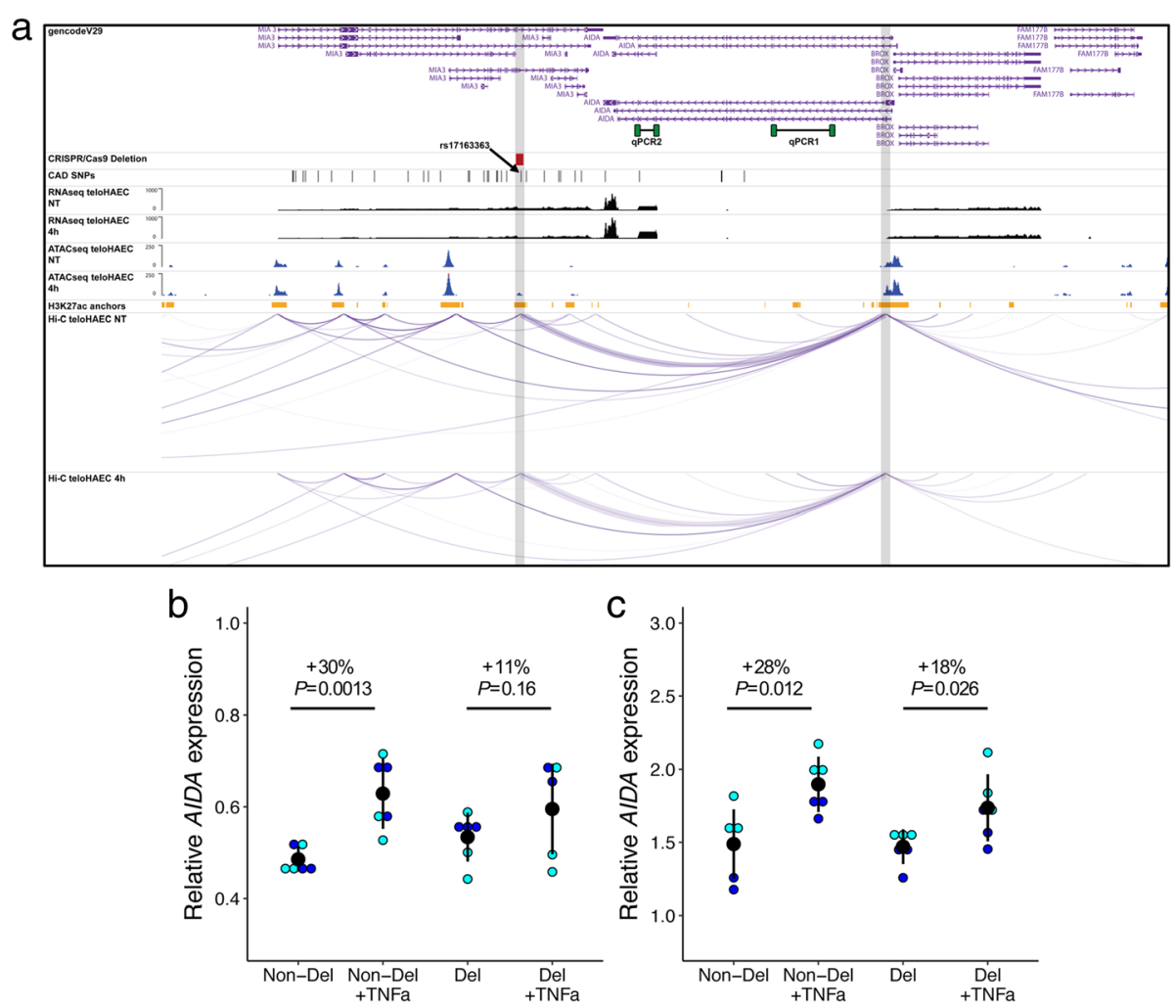

Fig. 5 AIDA upregulation by TNFa is controlled by a regulatory element that includes one coronary artery disease-associated SNP. a Graphical representation of the transcriptomic, epigenomic, and 3D conformation data at the AIDA coronary artery disease (CAD)-associated locus. The CRISPR/Cas9 deletion is indicated in red and both qPCR assays are represented in green. We added gray vertical bars to highlight the TNFa-sensitive open chromatin peak (left) and the AIDA promoter (right). To improve visualization, we also increased the width of the arcs linking both elements (purple). b Relative AIDA expression in teloHAEC without or with TNFa treatment for $4 \mathrm{~h}$ with QPCR assay \#1. Data was obtained from two independent experiments (circles in blue and cyan indicate the different biological replicates). For the non-deleted (Non-Del) and CRISPR/Cas9 heterozygote (Del) data points, we pooled data from three independent clones. Mean and standard deviation are plotted (black). For statistical analysis, we used linear regression correcting for batch effects and report two-tailed $P$ values. $\mathbf{c}$ As for $\mathbf{b}$, AIDA transcript quantification performed with GPCR assay \#2

To support our results, we tested one prediction by deleting a TNF $\alpha$-induced ATACseq open chromatin region in teloHAEC using CRISPR/Cas9. In heterozygous clones that carry this $\sim 1 \mathrm{~kb}$ deletion, the expression of $A I D A$ induced by TNFa treatment was strongly hindered (Fig. 5b, c). This is a promising result given that AIDA is differentially expressed in teloHAEC following $\mathrm{TNF} \alpha$ treatment $(\mathrm{NT}$ vs. $4 \mathrm{~h}, \mathrm{LFC}=0.49, \mathrm{FDR}=5.2 \times$ $10^{-19}$; Additional file 2) and the AIDA promoter interacts with the ATACseq peak as determined by $\mathrm{Hi}-\mathrm{C}$ (Additional file 11). This locus, defined by the sentinel GWAS variant rs67180937, is associated with CAD and includes 33 other variants in strong LD $\left(r^{2}>0.8\right.$ in European populations from the 1000 Genomes Project). Our deletion, however, only encompasses one of these 34 SNPs, rs17163363, which is an eQTL for AIDA in GTEx $\left(P=1.4 \times 10^{-6}\right)$. rs17163363 does not overlap perfectly with transcription factor binding motifs, although it is located 14 and 23 base pairs away, respectively, from NKX2-5 and MEF2A binding sites. MEF2 transcription factors have previously been implicated in CAD [27].
Despite several attempts, we failed to identify teloHAEC clones that are homozygous for the ATACseq peak deletion at the AIDA locus. This might indicate that baseline expression levels of AIDA, MIA3, and/or potentially other genes controlled by this regulatory element are essential for teloHAEC cell survival. An extension of this observation is that complete bi-allelic deletion of regulatory elements by CRISPR/Cas9, an approach now routinely attempted to functionally characterize GWAS loci, will often fail or generate negative results that are difficult to interpret. This highlights the importance to develop efficient and high-throughput protocols to combine genome editing and homologydirected repair to precisely replace candidate functional alleles in human cells [28]. Although rs17163363 is the only variant in $\mathrm{LD}$ with the CAD sentinel variant rs67180937 within the CRISPR/Cas9 deletion generated at the AIDA locus, we cannot conclude that it is causal as other unknown variants in the deleted region could mediate the effect on AIDA expression. To address the potential causal role of rs17163363 in CAD, we propose 
that an allele replacement experiment, potentially mediated by CRISPR/Cas9 homology-directed repair, is needed.

Our results implicate $A I D A$ in an inflammatory response that promotes atherosclerosis and CAD. Axin interaction partner and dorsalization antagonist, or $A I D A$, was first identified in a yeast-two-hybrid screen for interaction with the scaffold protein Axin [29]. AIDA homodimerizes but can also physically interact with NFKB inhibitor- $\alpha$ (NFKBIA) and TNF $\alpha$-induced protein 3 (TNFAIP3) [30], two genes that are highly overexpressed in teloHAEC following TNF $\alpha$ treatment (Additional file 2). In zebrafish, aida over-expression in embryos inhibits the dorsalizing activity of Axin by interfering with the activation of the c-Jun N-terminal kinase (JNK) [31]. JNK are multifunctional kinases that are activated by stresses and cytokines, including TNF $\alpha$, and that can control several cellular stress responses such as apoptosis [32]. In endothelial cells, JNK is also activated in response to pro-inflammatory stimuli [33]. Although it remains speculative, our data leads us to hypothesize that endothelial cell dysfunction mediated by the antagonizing effect of AIDA on JNK contributes to inter-individual variation in CAD risk in humans.

\section{Conclusions}

We anticipate that our integration map of vascular endothelial cell transcriptomic, epigenomic, and 3D conformation datasets, when combined with statistical finemapping of GWAS loci, will provide sufficient resolution to pinpoint causal variants and genes implicated in CAD and $\mathrm{BP} /$ hypertension. This map will allow further investigation into the roles that endothelial cell dysfunction plays in modulating the risk to develop these important chronic diseases. We illustrated our strategy by characterizing a TNF $\alpha$-responsive regulatory element that controls the expression of the novel CAD candidate gene $A I D A$. Encouragingly, a recent report identified another CAD-associated regulatory variant of PLPP3 that resides within a vascular endothelial enhancer activated by shear stress [34], suggesting that many CAD- and BPassociated variants may influence vascular endothelial phenotypes. Finally, our results underscore the critical importance of characterizing both resting and activated cells and lead us to propose a context-dependent, TNF $\alpha$ induced dysregulation of endothelial AIDA expression as a novel candidate mechanism for CAD.

\section{Methods}

\section{Cell culture}

Immortalized human aortic endothelial cells (teloHAEC) (ATCC, CRL-4052) were grown in vascular cell basal media (VCBM) (ATCC, PCS-100-030) supplemented with endothelial cell growth kit-VEGF (ATCC, PCS-100-
$041)$ and $200 \mathrm{U} / \mathrm{mL}$ penicillin and $200 \mu \mathrm{g} / \mathrm{mL}$ of streptomycin (ThermoFisher, 15140122). Primary human coronary artery endothelial cells (HCAEC) from a single male donor (ATCC, CC-2585) were grown in EGM$2 \mathrm{MV}$ (Lonza, CC-3202) supplemented with $200 \mathrm{U} / \mathrm{mL}$ penicillin and $200 \mu \mathrm{g} / \mathrm{mL}$ of streptomycin. TeloHAEC and $\mathrm{HCAEC}$ were maintained under a $5 \% \mathrm{CO}_{2}$ atmosphere at $37{ }^{\circ} \mathrm{C}$ and subcultured to $90 \%$ and $70-85 \%$ confluency, respectively. Both cell lines were used below three passages after thawing for all experiments.

\section{Endothelial dysfunction induction}

Endothelial cells were treated with concentrations ranging from 0.1 to $10 \mathrm{ng} / \mathrm{mL}$ of TNFa (PeproTech, 300$01 \mathrm{~A})$ prepared in culture media for $4 \mathrm{~h}$ and $24 \mathrm{~h}$ periods. Treatment with $10 \mathrm{ng} / \mathrm{mL}$ induced the most substantial endothelial dysfunction related alterations in both teloHAEC and HCAEC without significantly altering cell proliferation and viability. Two independent biological replicates of $10 \mathrm{ng} / \mathrm{mL}, 4 \mathrm{~h}$ only $(\mathrm{Hi}-\mathrm{C})$ or 4 and $24 \mathrm{~h}$ (RNAseq, ATACseq, ChIPseq) TNF $\alpha$ treatments for each cell line were used for data generation unless stated otherwise. Non-treated (NT) cells grown in parallel were used as control.

\section{RNA extraction and quantitative PCR}

TeloHAEC cells were seeded at $2 \times 10^{5}$ cells per well in 6-well plates, grown for 3 days (refreshed media at day 2) until reaching $95-100 \%$ confluency and subjected to TNF $\alpha$ treatment as described above. In order to guarantee the reliability and reproduction of the results, RNA extraction, cDNA synthesis, and qPCR experiments were conducted in accordance to the Minimum Information for Publication of Quantitative Real-Time PCR Experiments (MIQE) guidelines [35]. Total RNA was extracted using RNeasy Plus Mini kit (Qiagen) and analyzed with an RNA 6000 Nano kit (Agilent Technologies) to assess its concentration and integrity on an Agilent 2100 Bioanalyzer. Also, no contamination was found within RNA extracts as assessed by spectrophotometry using Take3 Micro-Volume plates (Biotek) or BioDrop $\mu$ Lite with absorbance ratio of $260 / 280 \mathrm{~nm}$ in a range of $2.0-2.15$ for all samples. cDNAs were then generated by reverse transcription from $1 \mu \mathrm{g}$ of total RNA (with RNA integrity number of 10 for all samples) using $1 \mathrm{U}$ of MultiScribe Reverse Transcriptase, $100 \mathrm{mM}$ dNTPS, $20 \mathrm{U}$ of RNase inhibitor and $1 \times$ Random Primers (Applied Biosystems, $4,374,966)$ in a $20 \mu \mathrm{L}$ volume reaction. Reverse transcription reaction was carried in three steps: $10 \mathrm{~min}$ at $25^{\circ} \mathrm{C}$, $120 \mathrm{~min}$ at $37^{\circ} \mathrm{C}$, and $5 \mathrm{~min}$ at $85^{\circ} \mathrm{C}$. qPCR reactions were set up with $1.25 \mu \mathrm{L}$ of $\mathrm{cDNA}$ (1/50 dilution based on dynamic range of previously done standard curve for all target genes), $5 \mu \mathrm{L}$ of Platinum SYBR Green qPCR SuperMix-UDG (ThermoFisher, 11733046), and $3.75 \mu \mathrm{L}$ 
of primer pair mix at $0.8 \mu \mathrm{M}$ each. qPCR reaction for each gene was performed in triplicates and carried out in a CFX384 Touch Real-Time PCR Detection System (Bio-Rad, 1855485) with the following thermal profile: 2 min at $50{ }^{\circ} \mathrm{C}, 15 \mathrm{~min}$ at $95^{\circ} \mathrm{C}$ and a three-step cycle of $10 \mathrm{~s}$ at $95^{\circ} \mathrm{C}, 15 \mathrm{~s}$ at $55^{\circ} \mathrm{C}$, and $15 \mathrm{~s}$ at $72{ }^{\circ} \mathrm{C}$ repeated 40 times. Following the amplification process, a melting curve analysis was performed to ensure the specificity of the amplified products. Also, resulting amplification products from previous qPCR standard curve experiments were run on $1 \%$ agarose gel and purified prior to Sanger sequencing in order to validate amplification of the desired target. To assert the absence of undesired contamination, $\mathrm{qPCR}$ reactions with no template controls for each gene were carried out simultaneously with no fluorescence detected. Cq values corresponding to the number of cycles to reach quantification threshold were determined with the CFX Manager 3.1 (Bio-Rad) software for all genes. Relative expression level for the axin interactor, dorsalization associated (AIDA) gene were calculated by the $\Delta \Delta C T$ method [36] normalized with the three reference genes glyceraldehyde 3-phosphate dehydrogenase $(G A P D H)$, hypoxanthine phosphoribosyltransferase 1 (HPRT1), and TATA-binding protein (TBP). Based on geNORM principles for accurate normalization of realtime quantitative RT-PCR data by geometric averaging of multiple internal control genes, a mean $M$ value always below 0.35 was generated from the GAPDH, HPRT1, and $T B P$ genes for all qPCR experiments. All primers were obtained from IDT Technologies. The primers sequences are listed in Additional file 15.

\section{RNAseq and differential gene expression analysis}

Stranded cDNA libraries prepared from quality-controlled RNA (see above) were sequenced using Illumina 100-bp paired-ends on a HiSeq 4000 platform, generating 50-60 million reads per condition per biological replicate. Reads were mapped to hg19 using hisat2 (http://ccb.jhu.edu/ software/hisat2/index.shtml). Samtools was used to sort the reads and convert to the BAM format. Transcripts were first identified for each sample, and then pooled together using stringtie (http://ccb.jhu.edu/software/stringtie/index.shtml). Transcript abundance was estimated by stringtie, and a fragments per kilobase of transcript per million (FPKM) count table was generated. Differential analysis of gene expression was performed using DESeq2 [37]. All possible comparisons for NT, TNFa $4 \mathrm{~h}$, and $24 \mathrm{~h}$ treatments were performed using the analysis of deviance function with default parameters. Genes with a false discovery rate (FDR, Benjamini \& Hochberg correction) < $0.1 \%$, and $\log _{10}$ fold-change $>0.3$ or $<-0.3$ in any of the 3 possible comparisons (NT vs. $4 \mathrm{~h}$; NT vs. $24 \mathrm{~h} ; 4 \mathrm{~h}$ vs. 24 h) were considered differentially expressed. Corresponding biological replicates output were merged using UCSC
BigWig and BigBed tools [38] for visualization purposes in the WashU Epigenome Browser [39].

\section{Assay for transposase-accessible chromatin with high throughput sequencing (ATACseq)}

TeloHAEC and HCAEC cells were seeded at $2 \times 10^{5}$ cells per well in 6-well plates, grown for 3 days (refreshed media at day 2) until reaching $95-100 \%$ confluency and subjected to TNF $\alpha$ treatment as described above. Adherent cells were detached using TrypsinEDTA (ATCC, PSC-999-003) and subsequently neutralized by Trypsin Neutralizing Solution (ATCC, PSC-999004). Following endothelial cell activation, ATACseq libraries were prepared as previously described [40] with the following specifications and modifications: $5 \times 10^{4}$ cells were spun down at $500 \mathrm{~g}$ for $5 \mathrm{~min}$ at $4{ }^{\circ} \mathrm{C}$. Whole cell pellets were subjected to a first round of cell membrane lysis using $50 \mu \mathrm{L}$ of ice-cold hypotonic buffer (0.1\% Sodium citrate tribasic dehydrate (Sigma-Aldrich, C8532); 0.1\% Triton X-100 (Sigma-Aldrich, X100)) and incubating on ice for $30 \mathrm{~min}$. The hypotonic buffer was removed by centrifugation at $500 \mathrm{~g}$ for $5 \mathrm{~min}$ at $4{ }^{\circ} \mathrm{C}$, and we subsequently discarded the supernatant. Crude nuclei lysates were prepared by resuspending cells in lysis buffer $(10 \mathrm{mM}$ Tris- $\mathrm{HCl} \mathrm{pH}$ 7.4 (Fisher Scientific, BP-1531); $10 \mathrm{mM} \mathrm{NaCl}$ (Fisher Scientific, BP-358-212); $3 \mathrm{mM}$ $\mathrm{MgCl}_{2}$ (Sigma-Aldrich, M8266); 0.1\% Igepal CA-630 (Sigma-Aldrich, I8896) and incubating for $30 \mathrm{~min}$ on ice. Following the removal of lysis buffer by centrifugation at $500 \mathrm{~g}$ for $5 \mathrm{~min}$ at $4{ }^{\circ} \mathrm{C}$, transposase reaction of open chromatin was achieved by resuspending free nuclei in tagmentation mix $(22.5 \mu \mathrm{L}$ Tagment DNA Buffer; $2.5 \mu \mathrm{L}$ Tagment DNA enzyme; $25 \mu \mathrm{L} \mathrm{H}_{2} \mathrm{O}$ ) (Illumina, FC-1211030) and incubating at $37^{\circ} \mathrm{C}$ for $30 \mathrm{~min}$. Purification of DNA was performed with MinElute (Qiagen, 28004) according to the manufacturer's protocol. Barcoding and amplification was prepared using Nextera Index Kit (Illumina, FC-121-1011) as previously described [40] with the following thermal profile: $30 \mathrm{~s}$ at $98^{\circ} \mathrm{C}$ and a three-step cycle of $10 \mathrm{~s}$ at $98^{\circ} \mathrm{C}, 30 \mathrm{~s}$ at $63^{\circ} \mathrm{C}$, and $1 \mathrm{~min}$ at $72{ }^{\circ} \mathrm{C}$ repeated 12 times followed by $5 \mathrm{~min}$ at $72{ }^{\circ} \mathrm{C}$. Amplified ATACseq libraries were purified using GeneRead Size Selection Kit (Qiagen, 180514) according to the manufacturer's protocol. Quality and quantity of final ATACseq libraries were assessed with the High Sensitivity DNA kit (Agilent, 5067-4626) ran on an Agilent 2100 Bioanalyzer. ATACseq libraries were sequenced using Illumina 125-bp paired-ends sequencing on a HiSeq 2500 platform with, generating between 38 and 43 million reads per condition per biological replicate.

ATAC library reads were processed through the ATACseq pipeline (https://github.com/kundajelab/atac_ dnase_pipelines). Adapters were removed using Cut- 
adapt. Reads were then mapped to hg19 using Bowtie2. Peak calling from BAM files was performed using MACS2 [41]. To create a "masterBED" peak file across conditions, peak files generated for each condition were merged using the merge function from BEDTools [42]. Mean scores from bedGraphs for each individual biological replicate were assigned to masterBED peak files using intersect (default parameters) and merge (-o mean) and used as input for differential analysis using DESeq2 [37]. All comparisons for NT, TNF $\alpha 4 \mathrm{~h}$, and $24 \mathrm{~h}$ treatments were performed using the analysis of deviance function with default parameters in DEseq2. ATACseq peaks with a false discovery rate (FDR, Benjamini \& Hochberg correction) $<0.1 \%$, and $\log _{10}$ fold-change $>0.3$ or $<-0.3$ in any of the 3 possible comparisons (NT vs. $4 \mathrm{~h}$; NT vs. $24 \mathrm{~h} ; 4 \mathrm{~h}$ vs. $24 \mathrm{~h}$ ) were considered differentially opened or closed. Corresponding biological replicates bedGraphs output from MACS were merged using UCSC BigWig and BigBed tools [36] for visualization purposes in the WashU Epigenome Browser [39]. For in silico footprinting, we used CENTIPEDE with default parameters [17]. For the enrichment analyses of CAD, $\mathrm{BP}$ and BMI SNPs in open chromatin regions, we retrieved sentinel variants from published large-scale GWAS $[8,11,21]$. We identified proxy variants in linkage disequilibrium $\left(r^{2}>0.8\right)$ using populations of European ancestry from the 1000 Genomes Project [43].

\section{Chromatin immunoprecipitation of H3K27 acetylation (H3K27ac) combined with high throughput sequencing (ChIPseq)}

TeloHAEC were seeded at $1.4 \times 10^{5}$ cells per $100 \mathrm{~mm}$ plates (1 plate per condition, 3 independent biological replicates), grown to $90-100 \%$ confluency (refreshed media every 2-3 days) and subjected to TNF $\alpha$ treatment as described above. Cells were washed with HBSS (Gibco, 14170161) and fixed in paraformaldehyde (PFA) $1 \%$ (Fisher Scientific, 15710) for $10 \mathrm{~min}$ at room temperature (RT). PFA was quenched in $134 \mathrm{mM}$ glycine for $5 \mathrm{~min}$ at RT. Fixed cells were washed with ice-cold PBS and collected with a cell scraper in ice-cold PBS. Cells were pelleted by centrifugation, washed in ice-cold PBS, and pelleted again before snap freezing in liquid nitrogen. Fixed cells were subject to lysis in $5 \mathrm{mM}$ PIPES$\mathrm{pH} 8.5,85 \mathrm{mM} \mathrm{KCl}, 1 \%(v / v)$ IGEPAL CA-630, $50 \mathrm{mM}$ NaF, $1 \mathrm{mM}$ PMSF, $1 \mathrm{mM}$ Phenylarsine Oxide, $5 \mathrm{mM}$ Sodium Orthovanadate and protease inhibitor cocktail (Sigma, 04693159001). Nuclei were then lysed in $50 \mathrm{mM}$ Tris- $\mathrm{HCl} \mathrm{pH}$ 8.0, $10 \mathrm{mM}$ EDTA, 1\% (w/v) SDS, $50 \mathrm{mM}$ NaF, $1 \mathrm{mM}$ PMSF, $1 \mathrm{mM}$ phenylarsine oxide, $5 \mathrm{mM}$ sodium orthovanadate and protease inhibitor cocktail. Chromatin immunoprecipitation was performed as previously described using $3.7 \mu \mathrm{g}$ of H3K27ac antibody (Diagenode; C15410196) per samples containing 500,
000 cells [44]. ChIPseq libraries were sequenced using Illumina 100-bp paired-end read sequencing on a NovaSeq 6000 instrument for approximately 150 million reads per sample. H3K27ac ChIPseq library raw reads were filtered for quality (phred33 $\geq 30)$ and length $(n \geq$ 32 ), and adapter sequences were removed using Trimmomatic [45]. Filtered reads were aligned to hg19 using BWA and peaks subsequently called using MACS2 [41] with non-IP input DNA as control. Corresponding bedGraphs output of biological replicates and input controls from MACS were merged using UCSC BigWig and BigBed tools [38] for visualization purposes in the WashU Epigenome Browser [39].

\section{In situ Hi-C library preparation and analysis}

TeloHAEC were seeded at $1.4 \times 10^{5}$ cells per $100 \mathrm{~mm}$ plates (4 plates per condition), grown to $90-100 \%$ confluency (refreshed media every 2-3 days) and subjected to the $4 \mathrm{~h}$ TNF $\alpha$ treatment as described above. In situ $\mathrm{Hi}-\mathrm{C}$ libraries were prepared as previously described [46] with the following specifications and modifications: approximately $8 \times 10^{6}$ cells per sample were crosslinked, pelleted and washed in ice-cold PBS prior to lysis and chromatin digestion with DpnII. Reverse crosslinking was performed in two subsequent 16 and $2 \mathrm{~h}$ incubations with $500 \mu \mathrm{g}$ of proteinase $\mathrm{K}$ prepared at $10 \mathrm{mg} / \mathrm{mL}$ in $5 \mathrm{mM}$ Tris- $\mathrm{HCl} \mathrm{pH} \mathrm{7.5,50 \%} \mathrm{glycerol,} 1 \mathrm{mM} \mathrm{CaCl} 2$ for each step. DNA purification was performed using $15 \mathrm{~mL}$ MaXtract High Density tubes (Qiagen, 129,065). PreNGS Hi-C DNA was quantified and quality-controlled with a DNA 7500 kit (Agilent, 5067-1506) ran on an Agilent 2100 Bioanalyzer. Prior to next-generation DNA sequencing (NGS), DNA extractions for quality control of chromatin integrity, digestion efficacy were performed with the following procedure: 1 volume of Phenol: Chloroform:Isoamyl Alcohol (25:24:1 v/v) (Invitrogen, 15593031) was added to lysate, vortexed and transferred to pre-spun Phase Lock Gel (VWR, 10847-800) and centrifuged for $5 \mathrm{~min}$ at $16,000 \mathrm{~g}$. The aqueous phase was kept, concentrated by speed-vacuum and subjected to $0.8 \%$ agarose gel electrophoresis. Quality-control 3CPCR of pre-NGS Hi-C libraries was performed in the ENr313 region using $800 \mathrm{ng}$ of template DNA, PfuUltra II Fusion HotStart DNA Polymerase (Agilent, 600672), $400 \mathrm{nM}$ ENr313_DpnII_Anchor1 primer \#1, $400 \mathrm{nM}$ ENr313_DpnII_Anchor1_Near primer \#2 and $250 \mu \mathrm{M}$ dNTPs with the following thermal profile: $2 \mathrm{~min}$ at $95^{\circ} \mathrm{C}$ and a three-step cycle of $30 \mathrm{~s}$ at $95^{\circ} \mathrm{C}, 30 \mathrm{~s}$ at $60^{\circ} \mathrm{C}$ and $30 \mathrm{~s}$ at $72^{\circ} \mathrm{C}$ repeated 35 times followed by $8 \mathrm{~min}$ at $72{ }^{\circ} \mathrm{C}$ [46]. For NGS preparation, between 20 and $40 \mu \mathrm{g}$ of purified Hi-C DNA was used as starting material for all subsequent steps. Sonication to 200-300 bp fragments was carried in an S2 Focused-ultrasonicator with no alterations to the suggested parameters. Biotin pulldown 
was performed with $200 \mu \mathrm{g}$ of Dynabeads MyOne Streptavidin C1 (Invitrogen, 65001) per sample. Production PCR was carried out with 9 cycles of PCR to obtain sufficient quantity for NGS while limiting PCR duplicates. Quality and quantity of final $\mathrm{Hi}-\mathrm{C}$ libraries were assessed on High Sensitivity DNA kit (Agilent, 5067-4626) ran on an Agilent 2100 Bioanalyzer. Final $\mathrm{Hi}-\mathrm{C}$ libraries were sequenced using Illumina 100-bp paired-ends sequencing on a Novaseq 6000, generating between 0.72 and 0.88 billion reads per condition per biological replicate.

$\mathrm{Hi}-\mathrm{C}$ reads were processed using the Juicer pipeline [47]. Hi-C libraries for all biological replicates had reads with the following quality measures: less than $10 \%$ below MAPQ threshold of 30 (average of 9.15\%), more than $62 \%$ intra-chromosomal interactions (average of 68.5\%) and less than $26 \%$ of inter-chromosomal interactions (average of 20.3\%). Correlation between biological replicates was assessed (Pearson's $r, 10 \mathrm{~kb}$ resolution $>0.95$; $50 \mathrm{~kb}$ resolution $>0.97 ; 100 \mathrm{~kb}$ resolution $>0.98$ ) before merging to increase statistical power. Contacts maps were normalized with Knight-Ruiz (KR) matrix balancing before all downstream analyses.

\section{A/B compartments calling and analysis}

Per chromosome principal component analysis (PCA) was performed by calling the eigenvector function from the Juicer pipeline using $50 \mathrm{~kb}$ resolution matrices with $\mathrm{KR}$ normalization. Using the $\mathrm{R}$ packages $\mathrm{TxDb}$ and Sushi, PC1 values were aligned to gene density in $50 \mathrm{~kb}$ windows. If needed, the sign of PC1 was adjusted to correlate positive $\mathrm{PC} 1$ values with gene-rich regions and negative PC1 with gene-poor regions. Contiguous bins of positive and negative $\mathrm{PC} 1$ were labeled as $\mathrm{A}$ and $\mathrm{B}$ compartments, respectively. Switching from A-to-B and B-to-A compartments upon TNF $\alpha$ treatment was retrieved from the differences in $\mathrm{A} / \mathrm{B}$ compartments called between NT and TNF $\alpha$-treated cells. Genes, ATACseq peaks, BP and CAD SNPs mapping to switching compartments were identified using map and merge functions from BEDTools with default parameters.

\section{Topologically associated domains (TADs) calling and analysis}

TAD calling was performed on teloHAEC (NT and $4 \mathrm{~h}$ TNF $\alpha$ ). KR normalized sparse matrices of $10 \mathrm{~kb}$ resolution were extracted from .hic files by calling the dump function from the Juicer pipeline [47]. TAD calling was performed using the Crane insulation score algorithm [48] Git version eecc2c9, with the default parameters (insulation delta span $=200 \mathrm{~kb}$, insulation square size $=500 \mathrm{~kb}$, insulation mode $=$ "mean," boundary margin of error $=3$, noise threshold $=0.1$ ). TADs that overlap with the centromeres, as well as regions at either end of each chromosome, were excluded from analyses. To determine if a TAD boundary overlapped with a feature (e.g., SNPs, ChIPseq, TSS, enhancer, promoter), we added a $10 \mathrm{~kb}$ outward buffer to the boundary coordinates. To determine if TADs were stable or changed following TNF $\alpha$ treatment, we added a 20-kb outward buffer to the boundary coordinates. The physical distance of CAD and BP sentinel SNPs with the closest TAD boundary was compared with the distance of control SNPs matched based on minor allele frequency, gene density, gene proximity and the number of LD proxies using SNPsnap default parameters [49]. To derive empirical $P$ values, we considered the median distances to the closest TAD boundary of 100 sets of matched SNPs and compared them to the median distance of the CAD or BP SNPs.

\section{Loop calling between regulatory regions and promoters}

$\mathrm{Hi}-\mathrm{C}$ reads were processed using the $\mathrm{HiC}$-Pro pipeline (https://github.com/nservant/HiC-Pro). hichipper (https:// github.com/aryeelab/hichipper) was used to call loops between promoters and ATACseq peaks that harbor CAD or BP GWAS SNPs. Gene promoters' coordinates were downloaded from the EPDnew database (https://epd.vitalit.ch/human/human_database.php). The detailed steps used to integrate and combine the GWAS, RNAseq, ATACseq, Hi-C, and GTEx data are provided in Additional file 11.

\section{CRISPR/Cas9 genome editing}

Pairs of guide RNAs (sgRNAs) were designed for each targeted genomic deletion and cloned into the pHKO9 vector under control of the same U6 promoter (Additional file 16). HEK $293 \mathrm{~T}$ cells were seeded at $5 \times 10^{5}$ cells/well in 6-well plates for $24 \mathrm{~h}$. Lentivirus were produced by co-transfecting the envelope and packaging plasmids pMD2G and psPAX2 respectively with the dual sgRNA expressing pHKO9 vector in HEK $293 \mathrm{~T}$ cells using Lipofectamine 2000 (ThermoFisher, 11,668,027) for $4 \mathrm{~h}$ then switched to virus-producing media containing $10 \mu \mathrm{g} / \mathrm{mL}$ of BSA. Viral supernatant was harvested $48 \mathrm{~h}$ and $72 \mathrm{~h}$ following transfection and filtered through $0.45 \mu \mathrm{m}$ filters. TeloHAEC cells stably expressing an active Cas9 protein were seeded at $2 \times 10^{5}$ cells/well in 6well plates and later infected with the virus preparation and media containing $0.7 \mu \mathrm{g} / \mathrm{mL}$ of polybrene (Sigma, H9268). Selection with $200 \mu \mathrm{g} / \mathrm{mL}$ of G418 (Fisher, MT30234CR) was started $48 \mathrm{~h}$ post-infection. Antibiotic selective pressure was maintained for 5-6 days or until non-infected cells were dead. Sub-populations of 50 cells were derived and screened via PCR using primers surrounding the expected deletion (out-out PCR) (Additional file 16). Clonal cell lines were then derived from a PCR-positive deletion sub-population. Another round of out-out PCR was performed on these select clonal cell lines and PCR products were purified and cloned into 
pDrive cloning vector system (Qiagen, 231122) or into the pUC19 vector using In-Fusion HD cloning system (Takara, 638,909). Genotypes of all possible alleles were confirmed by gel electrophoresis (Additional file 14) and Sanger sequencing. Select genome reengineered clones were then seeded at $9 \times 10^{4}$ cells per well in 12-well plates, grown to $90-100 \%$ confluency (refreshed media every 2-3 days) and subjected to a 4-h TNF $\alpha$ treatment as described above. Total RNA was then extracted, quantified, quality controlled and reverse transcribed as described above. qPCR was performed for the target gene anchored at the receiving end of the chromatin loop with 2 different primer pairs capturing exons in either 5' or 3' of AIDA.

\section{Additional files}

Additional file 1: Comparison of RNA-sequencing ( $\mathrm{A}$ and $\mathrm{B}$ ) and ATACsequencing $(C$ and $D)$ results in teloHAEC non-treated (NT) or treated with TNFa for $4 \mathrm{~h}$ or $24 \mathrm{~h}$. (DOCX $377 \mathrm{~kb}$ )

Additional file 2: RNA-sequencing results of teloHAEC NT or treated with TNFalpha for 4 or $24 \mathrm{~h}$. (XLSX $216 \mathrm{~kb}$ )

Additional file 3: Biological pathways enriched for genes that are differentially expressed in teloHAEC upon TNFa treatment. (XLSX $14 \mathrm{~kb}$ )

Additional file 4: ATAC-sequencing results of teloHAEC NT or treated with TNFa for 4 or $24 \mathrm{~h}$. (XLSX $381 \mathrm{~kb}$ )

Additional file 5: Log10-fold-change (LFC) of ATACseq peaks comparing non-treated vs. $4 \mathrm{~h}$ TNFa-treated teloHAEC. (DOCX $315 \mathrm{~kb}$ )

Additional file 6: Transcription factor binding motifs that are represented in ATACseq peaks profiled in NT or TNFa-treated teloHAEC. (XLSX $16 \mathrm{~kb})$

Additional file 7: Biological pathways that are enriched for transcription factors that bind within ATACseq peaks profiled in NT and TNFa treated teloHAEC. (XLSX $14 \mathrm{~kb}$ )

Additional file 8: Enrichment of coronary artery disease (CAD)- and blood pressure (BP)-associated SNPs (or their linkage disequilibrium proxies) in teloHAEC ATACseq or H3K27ac ChIPseq peaks. (XLSX $10 \mathrm{~kb}$ )

Additional file 9: Transcription factor (TF) binding motifs located in ATACseq peaks that overlap with SNPs associated with coronary artery disease (CAD) or blood pressure (BP). (XLSX $61 \mathrm{~kb}$ )

Additional file 10: Topologically associated domains (TADs) in unstimulated teloHAEC endothelial cells. (DOCX $854 \mathrm{~kb}$ )

Additional file 11: $\mathrm{Hi}-\mathrm{C}$ loops between open chromatin regions and gene promoters in teloHAEC. The regulatory elements were defined using ATACseq data. (XLSX $187 \mathrm{~kb}$ )

Additional file 12 Definition of the headers used in Additional file 11. (XLSX $9 \mathrm{~kb}$ )

Additional file 13: Detailed steps to generate and integrate the GWAS SNP, RNAseq, ATACseq, eQTL, and HiC data. (XLSX 9 kb)

Additional file 14: Validation of the CRISPR/Cas9-induced deletion at the AIDA locus (DOCX $790 \mathrm{~kb}$ )

Additional file 15: Primers sequences for quantitative PCR. (XLSX $10 \mathrm{~kb}$ ) Additional file 16: Primers used to generate indels in teloHAEC. (XLSX 8 kb)

Additional file 17: Review history. (DOCX $323 \mathrm{~kb}$ )

\section{Abbreviations}

ATACseq: Assay for transposase-accessible chromatin with high-throughput sequencing: BMI: Body mass index; BP: Blood pressure; CAD: Coronary artery disease; ChIPseq: Chromatin immunoprecipitation with high-throughput sequencing; DE: Differentially expressed; DO: Differentially opened and/or closed; eQTL: Expression quantitative trait locus; FDR: False discovery rate; GWAS: Genome-wide association study; H3K27ac: Acetylation of lysine-27 on histone 3; HCAEC: Human coronary artery endothelial cells; Hi-C: Chromatin conformation capture with high-throughput sequencing; HUVEC: Human umbilical vein endothelial cells; LD: Linkage disequilibrium; LFC: Log $_{10}$ foldchange; NT: Non-treated; RNAseq: Ribonucleic acid sequencing; SNP: Single nucleotide polymorphism; TAD: Topologically associated domain; teloHAEC: Immortalized human aortic endothelial cells; TNFa: Tumor necrosis factor-a; TSS: Transcription start site

\section{Acknowledgements}

We thank the members of our laboratories for comments and Albena Pramatarova for scientific discussions and technical assistance.

\section{Review history}

The review history is available at Additional file 17.

\section{Authors' contributions}

SL, V-ACF, and GL conceived and designed the experiments. SL, V-ACF, $S M d B, F L, M B, M-M S, R D, T K$, and KSL performed the experiments. TP and GL secured funding. All authors analyzed the results. SL, SMdB, KSL, and GL wrote the manuscript with contributions from all authors. All authors read and approved the final manuscript.

\section{Funding}

This work was funded by the Canadian Institutes of Health Research (MOP \#136979), the Heart and Stroke Foundation of Canada (Grant \#G-18-0021604), the Canada Research Chair Program, and the Montreal Heart Institute Foundation (to GL). This work was also supported by the NIH Common Fund Program, grant U01CA200147, as a Transformative Collaborative Project Award (TCPA) TCPA-2017-PASTINEN / CIHR NTC-154083.

\section{Availability of data and materials}

The datasets generated and/or analyzed during the current study are available in the NCBI's Gene Expression Omnibus (GEO) repository (GSE126200) [50].

\section{Ethics approval and consent to participate}

Not applicable. Experiments were performed with commercially available cell lines.

\section{Consent for publication}

Not applicable.

\section{Competing interests}

The authors declare that they have no competing interests.

\section{Author details}

${ }^{1}$ Montreal Heart Institute, 5000 Belanger street, Montréal, Québec H1T 1C8, Canada. ${ }^{2}$ Faculté de Médecine, Université de Montréal, Montréal, Québec H3T 1J4, Canada. ${ }^{3}$ McGill University and Genome Québec Innovation Center, Montréal, Québec H3A 0G1, Canada. ${ }^{4}$ Center for Pediatric Genomic Medicine (CPGM), Children's Mercy Kansas City, 2401 Gillham Road, Kansas City, MO 64108, USA

Received: 14 February 2019 Accepted: 27 June 2019

Published online: 08 July 2019

\section{References}

1. Nelson MR, Tipney H, Painter JL, Shen J, Nicoletti P, Shen Y, et al. The support of human genetic evidence for approved drug indications. Nat Genet. 2015;47(8):856-60.

2. Pers TH, Karjalainen JM, Chan Y, Westra HJ, Wood AR, Yang J, et al. Biological interpretation of genome-wide association studies using predicted gene functions. Nat Commun. 2015:6:5890

3. Trynka G, Sandor C, Han B, Xu H, Stranger BE, Liu XS, et al. Chromatin marks identify critical cell types for fine mapping complex trait variants. Nat Genet. 2012:45:124-30 
4. Finucane HK, Bulik-Sullivan B, Gusev A, Trynka G, Reshef Y, Loh PR, et al. Partitioning heritability by functional annotation using genome-wide association summary statistics. Nat Genet. 2015;47(11):1228-35.

5. Musunuru K, Strong A, Frank-Kamenetsky M, Lee NE, Ahfeldt T, Sachs KV, et al. From noncoding variant to phenotype via SORT1 at the 1p13 cholesterol locus. Nature. 2010;466(7307):714-9.

6. Bauer DE, Kamran SC, Lessard S, Xu J, Fujiwara Y, Lin C, et al. An Erythroid enhancer of BCL11A subject to genetic variation determines fetal hemoglobin level. Science. 2013;342:253-7.

7. Lessard S, Gatof ES, Beaudoin M, Schupp PG, Sher F, Ali A, et al. An erythroid-specific ATP2B4 enhancer mediates red blood cell hydration and malaria susceptibility. J Clin Invest. 2017;127(8):3065-74.

8. van der Harst P, Verweij N. Identification of 64 novel genetic loci provides an expanded view on the genetic architecture of coronary artery disease. Circ Res. 2018;122(3):433-43.

9. Klarin D, Zhu QM, Emdin CA, Chaffin M, Horner S, McMillan BJ, et al. Genetic analysis in UK Biobank links insulin resistance and transendothelial migration pathways to coronary artery disease. Nat Genet. 2017;49(9):1392-7.

10. Howson JMM, Zhao W, Barnes DR, Ho WK, Young R, Paul DS, et al. Fifteen new risk loci for coronary artery disease highlight arterial-wall-specific mechanisms. Nat Genet. 2017;49(7):1113-9.

11. Evangelou E, Warren HR, Mosen-Ansorena D, Mifsud B, Pazoki R, Gao H, et al. Genetic analysis of over 1 million people identifies 535 new loci associated with blood pressure traits. Nat Genet. 2018;50(10):1412-25.

12. Warren HR, Evangelou E, Cabrera CP, Gao H, Ren M, Mifsud B, et al. Genome-wide association analysis identifies novel blood pressure loci and offers biological insights into cardiovascular risk. Nat Genet. 2017;49(3):40315.

13. Khera AV, Kathiresan S. Genetics of coronary artery disease: discovery, biology and clinical translation. Nat Rev Genet. 2017;18(6):331-44.

14. Cahill PA, Redmond EM. Vascular endothelium - gatekeeper of vessel health. Atherosclerosis. 2016;248:97-109.

15. Fairfax BP, Humburg P, Makino S, Naranbhai V, Wong D, Lau E, et al. Innate immune activity conditions the effect of regulatory variants upon monocyte gene expression. Science. 2014;343(6175):1246949.

16. Zhang H, Park Y, Wu J, Chen X, Lee S, Yang J, et al. Role of TNF-alpha in vascular dysfunction. Clin Sci (Lond). 2009;116(3):219-30.

17. Raj A, Shim H, Gilad Y, Pritchard JK, Stephens M. msCentipede: modeling heterogeneity across genomic sites and replicates improves accuracy in the inference of transcription factor binding. PLoS One. 2015;10(9):e0138030.

18. Roadmap Epigenomics C, Kundaje A, Meuleman W, Ernst J, Bilenky M, Yen $A$, et al. Integrative analysis of 111 reference human epigenomes. Nature. 2015;518(7539):317-30.

19. Maurano MT, Humbert R, Rynes E, Thurman RE, Haugen E, Wang H, et al. Systematic localization of common disease-associated variation in regulatory DNA. Science. 2012;337(6099):1190-5.

20. Lo KS, Vadlamudi S, Fogarty MP, Mohlke KL, Lettre G. Strategies to fine-map genetic associations with lipid levels by combining epigenomic annotations and liver-specific transcription profiles. Genomics. 2014;104(2):105-12.

21. Locke AE, Kahali B, Berndt SI, Justice AE, Pers TH, Day FR, et al. Genetic studies of body mass index yield new insights for obesity biology. Nature. 2015;518(7538):197-206.

22. Schmidt EM, Zhang J, Zhou W, Chen J, Mohlke KL, Chen YE, et al. GREGOR: evaluating global enrichment of trait-associated variants in epigenomic features using a systematic, data-driven approach. Bioinformatics. 2015; 31(16):2601-6.

23. Lieberman-Aiden E, Berkum NLV, Williams L, Imakaev M, Ragoczy T, Telling $A$, et al. Comprehensive mapping of long-range interactions reveals folding principles of the human genome. Science. 2009;326:289-94.

24. Dixon JR, Gorkin DU, Ren B. Chromatin domains: the unit of chromosome organization. Mol Cell. 2016;62(5):668-80.

25. Dixon JR, Selvaraj S, Yue F, Kim A, Li Y, Shen Y, et al. Topological domains in mammalian genomes identified by analysis of chromatin interactions. Nature. 2012:485(7398):376-80

26. Consortium G. Human genomics. The Genotype-Tissue Expression (GTEx) pilot analysis: multitissue gene regulation in humans. Science. 2015; 348(6235):648-60.

27. Beaudoin M, Gupta RM, Won HH, Lo KS, Do R, Henderson CA, et al. Myocardial infarction-associated SNP at 6p24 interferes with MEF2 binding and associates with PHACTR1 expression levels in human coronary arteries. Arterioscler Thromb Vasc Biol. 2015;35(6):1472-9.
28. Sharon E, Chen SA, Khosla NM, Smith JD, Pritchard JK, Fraser HB. Functional genetic variants revealed by massively parallel precise genome editing. Cell. 2018;175(2):544-57 e16.

29. Rui HL, Fan E, Zhou HM, Xu Z, Zhang Y, Lin SC. SUMO-1 modification of the C-terminal KVEKVD of Axin is required for JNK activation but has no effect on Wnt signaling. J Biol Chem. 2002;277(45):42981-6.

30. Huttlin EL, Bruckner RJ, Paulo JA, Cannon JR, Ting L, Baltier K, et al. Architecture of the human interactome defines protein communities and disease networks. Nature. 2017;545(7655):505-9.

31. Rui $Y, X u Z$, Xiong $B, C a o ~ Y$, Lin $S$, Zhang $M$, et al. A beta-cateninindependent dorsalization pathway activated by Axin/JNK signaling and antagonized by aida. Dev Cell. 2007;13(2):268-82.

32. Cui J, Zhang $M$, Zhang YQ, Xu ZH. JNK pathway: diseases and therapeutic potential. Acta Pharmacol Sin. 2007;28(5):601-8.

33. Chen X, Lin J, Hu T, Ren Z, Li L, Hameed I, et al. Galectin-3 exacerbates oxLDL-mediated endothelial injury by inducing inflammation via integrin beta1-RhoA-JNK signaling activation. J Cell Physiol. 2019;234(7):1099011000. https://doi.org/10.1002/jcp.27910.

34. Krause MD, Huang RT, Wu D, Shentu TP, Harrison DL, Whalen MB, et al. Genetic variant at coronary artery disease and ischemic stroke locus 1p32.2 regulates endothelial responses to hemodynamics. Proc Natl Acad Sci U S A. 2018:115(48):E11349-E58.

35. Bustin SA, Benes V, Garson JA, Hellemans J, Huggett J, Kubista M, et al. The MIQE guidelines: minimum information for publication of quantitative realtime PCR experiments. Clin Chem. 2009;55(4):611-22.

36. Schmittgen TD, Livak KJ. Analyzing real-time PCR data by the comparative C(T) method. Nat Protoc. 2008;3(6):1101-8.

37. Love Ml, Huber W, Anders S. Moderated estimation of fold change and dispersion for RNA-seq data with DESeq2. Genome Biol. 2014;15(12):550.

38. Kent WJ, Zweig AS, Barber G, Hinrichs AS, Karolchik D. BigWig and BigBed: enabling browsing of large distributed datasets. Bioinformatics. 2010;26(17): 2204-7.

39. Zhou X, Maricque B, Xie M, Li D, Sundaram V, Martin EA, et al. The human Epigenome browser at Washington University. Nat Methods. 2011;8(12):989-90.

40. Buenrostro JD, Wu B, Chang HY, Greenleaf WJ. ATAC-seq: a method for assaying chromatin accessibility genome-wide. Curr Protoc Mol Biol. 2015; 109:21 9 1-9.

41. Zhang Y, Liu T, Meyer CA, Eeckhoute J, Johnson DS, Bernstein BE, et al. Model-based analysis of ChIP-Seq (MACS). Genome Biol. 2008;9(9):R137.

42. Quinlan AR, Hall IM. BEDTools: a flexible suite of utilities for comparing genomic features. Bioinformatics. 2010;26(6):841-2.

43. Genomes Project $C$, Abecasis GR, Auton A, Brooks LD, DePristo MA, Durbin $\mathrm{RM}$, et al. An integrated map of genetic variation from 1,092 human genomes. Nature. 2012:491(7422):56-65.

44. Cheung WA, Shao X, Morin A, Siroux V, Kwan T, Ge B, et al. Functional variation in allelic methylomes underscores a strong genetic contribution and reveals novel epigenetic alterations in the human epigenome. Genome Biol. 2017;18(1):50

45. Bolger AM, Lohse M, Usadel B. Trimmomatic: a flexible trimmer for Illumina sequence data. Bioinformatics. 2014;30(15):2114-20.

46. Belaghzal H, Dekker J, Gibcus JH. Hi-C 2.0: an optimized Hi-C procedure for high-resolution genome-wide mapping of chromosome conformation. Methods. 2017;123:56-65.

47. Durand NC, Shamim MS, Machol I, Rao SS, Huntley MH, Lander ES, et al. Juicer provides a one-click system for analyzing loop-resolution $\mathrm{Hi}-\mathrm{C}$ experiments. Cell Syst. 2016;3(1):95-8.

48. Crane E, Bian Q, McCord RP, Lajoie BR, Wheeler BS, Ralston EJ, et al. Condensin-driven remodelling of $X$ chromosome topology during dosage compensation. Nature. 2015;523(7559):240-4.

49. Pers TH, Timshel P, Hirschhorn JN. SNPsnap: a web-based tool for identification and annotation of matched SNPs. Bioinformatics. 2015:31(3):418-20.

50. Lalonde S, Codina-Fauteux V-A, Méric de Bellefon S, Leblanc F, Beaudoin M, Simon M-M, et al., Integrative analysis of vascular endothelial cell genomic features identifies AIDA as a coronary artery disease candidate gene. NCBI Gene Expression Omnibus (GEO). Accession GSE126200. https://www.ncbi. nlm.nih.gov/geo/query/acc.cgi?acc=GSE126200.

\section{Publisher's Note}

Springer Nature remains neutral with regard to jurisdictional claims in published maps and institutional affiliations. 